\title{
EDITORIAL
}

\section{Breast cancer screening and mortality}

T he furor over breast cancer screening is largely because there is a lack of randomized trials that have all-cause mortality as their end point. Such data would settle the issue of whether or not harm is done to people who undergo routine screening, who do not have cancer, but might be at a greater risk of morbidity and dying because of repetitive biopsies and unnecessary surgery. All-cause mortality studies require large numbers of participants and are expensive and are not likely to be done in the future either. The National Cancer Institute (NCI) had one chance to do such a study when the Cancer Control Program was established after the passage of The National Cancer Act in 1971. The NCI sponsored mammography screening in a group of 250,000 women (a large enough number for an all-cause mortality study) but, despite the outcry from critics, it neglected to design the program as a test of the risks versus benefits of routine mammography. The NCI had already bought into the necessity of routine screening of all women who are aged 40 years or older. It was a great waste of money and an embarrassment to the NCI. Furthermore, approaches to screening are changing so much at present that any study designed to address these questions would be out of date by the time it was completed anyhow.

Thus, on the one hand we are stuck with unsettled arguments about the potential negative impact of routine screening on the risk of dying and quality of life, and on the other hand the saving of the lives of a few at the expense of screening many. We simply don't have the data and should admit it. In fact, we should have admitted it long ago. However, each side, pro or con routine screening, is so wrapped up in their belief they are right, that communication of recommendations to the public is flawed. I think the US Preventive Service Task Force (USPSTF) was clumsy in how they handled their conclusions. They were trying to show what good scientists they were. To the public, the phrase "There are no data to support" means you shouldn't do it. To the USPSTF it should have meant, we have no real basis to make a recommendation. So, there is no way to make an accurate recommendation for screening under the age of 50 or at 1-year or 2-year intervals. If women know this they can decide, with the help of their doctors, whether or not to undergo screening.

The epitome of this silliness is illustrated over the recommendations for breast self examination or breast examination by a physician. There has never been and never will be any data to prove it works to reduce overall mortality from breast cancer for the reason cited above. Nonetheless, women do on occasion pick up early lesions that might save their lives so why shouldn't they examine their breasts in an organized way. It costs nothing. What is more, if physicians follow the USPSTF recommendation they should not include an exam of the breasts in a routine physical exam either. How silly is that? First, patients and doctors alike will pick up early lesions that might, at a minimum, alter the nature of treatment in the patient's favor. Second, a physical exam is a physical exam and a good physician will examine the entire body. Gail Collins a columnist at the Washington Post, who wrote about the USPSTF controversy quipped, appropriately enough, that if we follow the USPSTF recommendations "a woman's breasts should never be touched except in anticipation of sex". I think she captured the silliness of their recommendation in that phrase. We simply cannot be sure that altering the current screening recommendations for breast cancer will benefit anyone. It then simply becomes an issue, no matter who may say it isn't, of who pays for it. And that's a separate debate. But, in my view, a woman who wants the security of screening by mammography should not be denied it.

doi:10.1038/nrclinonc.2009.232

\author{
4 We \\ simply cannot \\ be sure that \\ altering \\ the current \\ screening \\ recommen- \\ dations \\ will benefit \\ anyone 77
}

